\title{
Implementation of Multiple-Valued CAM Functions by LUT Cascades
}

\author{
Tsutomu Sasao \\ Department of Computer Science \\ and Electronics \\ Kyushu Institute of Technology \\ Iizuka, 820-8502 JAPAN
}

\author{
Jon T. Butler \\ Department of Electrical \\ and Computer Engineering \\ Naval Postgraduate School \\ Monterey, CA, 93943-5121 U.S.A.
}

\begin{abstract}
In this paper, we introduce three types of multiplevalued content-addressable memories (CAMs): ordinary CAMs (CAMs), distance d CAMs, and ${ }^{*} C A M s$. Ordinary CAMs require an exact match, while ${ }^{*} C A M s$ allow wildcard matches. In a distance d CAM, a match occurs even if at most d digits differ. Then, we define multiple-valued CAM functions represented by these CAMs. Next, we show an approach to realize each CAM function by an LUT cascade, which is a series connection of RAMs. Experimental results for both two-valued and multi-valued cases are shown.
\end{abstract}

\section{Introduction}

Ordinary memory produces the data at a given address. Conversely, a content-addressable memory (CAM) [5] produces the address of the given data. If the given data does not exist anywhere in the CAM, a special address (e.g. $00 \ldots 0)$ is produced. CAMs are often used in pattern matching applications, since they are much faster than software implementations. Other applications include routers for the internet $[2,4,6,15]$, processor caches, translation lookaside buffers (TLB), data compression applications, database accelerators, and neural networks. Several implementations of multiple-valued CAMs have been proposed [3, 14].

$p$-valued CAMs store $p$-nary vectors. We consider three types of $p$-valued CAMs: ordinary CAM (CAM), distance $d$ CAMs, and $*$ CAM. CAMs produce an address only if there is an exact match to the input data. A distance $d$ CAM is similar to a CAM except that there can be $d$ or fewer mismatches. Distance $d$ CAMs are used in pattern matching applications. A *CAM is similar to a CAM except there can be a mismatch only in positions designated by a $*$. Therefore, a binary *CAM stores a three valued vector, where each element is chosen from $\{0,1, *\}^{1}$. Such CAMs are

\footnotetext{
$1 *$ CAMs are sometimes called ternary CAMs or TCAM[6].
}

often used in routers for the internet.

We present a method to implement a CAM function by an LUT cascade, which is a series connection of RAMs. We show that this results in a simple implementation. The LUT cascade uses ordinary RAMs instead of special semiconductor CAMs, and show significant promise in reducing power dissipation, which is the problem in existing CAMs $[9,15]$.

Example 1.1 Consider an e-mail system in a company with 50,000 employees. Each employee has an employee number consisting of 7 digits. Also, each employee has a login name of an e-mail account consisting of 8 letters from the 26letter English alphabet.

CAM: Consider a table that yields the employee number from a given login name. Since each letter can be represented by 5 bits, the table has $5 \times 8=40$ binary inputs and $\left\lceil\log _{2} 10^{7}\right\rceil=24$ binary outputs. A direct implementation by a single conventional memory requires $2^{40} \times 24=$ $2.6 \times 10^{13}$ bits, which is 3 terabytes. Although there exist $27^{8} \cong 2.82 \times 10^{11}$ different login names (each consisting of 26 letters and a space), only 50,000 are used. We can use a CAM to greatly reduce the memory needed by the direct implementation.

Distance 1 CAM: The E-mail administrator sometimes wants to know the correct login name from an incorrect login name. Suppose that the correct login name is MORAGA. However, due to the smudged FAX or an unclear handwritten document, the login name may appear as MOROGA or MARAGA or MORAGQ. It would be convenient if the system showed the correct login name when there is no em-

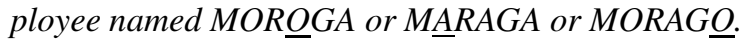

*CAM: The E-mail administrator wants to know the correct login name from an uncertain login name. Suppose that the administrator knows only a part of the login name: ***KOWSKI, for example, where * corresponds to any letter. It would be convenient if the system can show the possible correct login names, such as PERKOWSKI and FALKOWSKI.

(End of Example) 
Table 2.2. CAM function truth table

Table 2.1. CAM

Table

\begin{tabular}{|c|c|}
\hline Address & Vectors \\
\hline 1 & 0010 \\
\hline 2 & 0111 \\
\hline 3 & 1101 \\
\hline 4 & 0101 \\
\hline 5 & 0011 \\
\hline 6 & 1011 \\
\hline 7 & 0001 \\
\hline
\end{tabular}

\begin{tabular}{|cccc|ccc|}
\hline$x_{1}$ & $x_{2}$ & $x_{3}$ & $x_{4}$ & $f_{2}$ & $f_{1}$ & $f_{0}$ \\
\hline 0 & 0 & 0 & 0 & 0 & 0 & 0 \\
0 & 0 & 0 & 1 & 1 & 1 & 1 \\
0 & 0 & 1 & 0 & 0 & 0 & 1 \\
0 & 0 & 1 & 1 & 1 & 0 & 1 \\
0 & 1 & 0 & 0 & 0 & 0 & 0 \\
0 & 1 & 0 & 1 & 1 & 0 & 0 \\
0 & 1 & 1 & 0 & 0 & 0 & 0 \\
0 & 1 & 1 & 1 & 0 & 1 & 0 \\
1 & 0 & 0 & 0 & 0 & 0 & 0 \\
1 & 0 & 0 & 1 & 0 & 0 & 0 \\
1 & 0 & 1 & 0 & 0 & 0 & 0 \\
1 & 0 & 1 & 1 & 1 & 1 & 0 \\
1 & 1 & 0 & 0 & 0 & 0 & 0 \\
1 & 1 & 0 & 1 & 0 & 1 & 1 \\
1 & 1 & 1 & 0 & 0 & 0 & 0 \\
1 & 1 & 1 & 1 & 0 & 0 & 0 \\
\hline
\end{tabular}

\section{Content Addressable Memory}

We adopt the following definitions, which are intended to model certain physical devices ${ }^{2}$.

\section{$2.1 \quad \mathrm{CAM}$}

Definition 2.1 An n-input, m-output, p-valued, k-entry CAM table stores $k$-element $p$-nary vectors at addresses 1 through $k$. An address is an m-element p-nary vector, where $m=\left\lceil\log _{p}(k+1)\right\rceil$. Let $P=\{0,1, \ldots, p-1\}$. The corresponding CAM function is a logic function $\vec{f}: P^{n} \rightarrow P^{m}$, where $\vec{f}(\vec{x})$ is the address of the entry that matches $\vec{x}$ exactly. If no such entry exists, $\vec{f}(\vec{x})=0^{m}$.

Example 2.1 Consider the CAM table shown in Table 2.1. Here, $n=4, m=3, p=2$, and $k=7$. The truth table of the CAM function is shown in Table 2.2. The function's output is an address if there is an exact match (e.g., $\vec{f}(1,1,0,1)=$ 011). If the input vector is not stored in the CAM table, the output is 000 (e.g., $\vec{f}(1,1,1,0)=000) . \quad$ (End of Example)

\subsection{Distance $d$ CAM}

A distance $d$ CAM is used for pattern recognition and electronic dictionary $[7,8]$ applications.

Definition 2.2 Given a CAM table, the corresponding distance d CAM function is a logic function $\vec{f}: P^{n} \rightarrow P^{m}$, where $\vec{f}(\vec{x})$ is the address of a CAM table entry that differs

\footnotetext{
${ }^{2}$ Some CAMs [1] have both Address and Match Flag outputs. In this case, the vectors are stored from address 0 (instead of 1), and MatchFlag= 1 if at least one element in the CAM table matches. Our CAM is different from such CAMs.
}

Table 2.3. Distance 1 CAM function truth table

\begin{tabular}{|cccc|ccc|}
\hline$x_{1}$ & $x_{2}$ & $x_{3}$ & $x_{4}$ & $f_{2}$ & $f_{1}$ & $f_{0}$ \\
\hline 0 & 0 & 0 & 0 & 0 & 0 & 1 \\
0 & 0 & 0 & 1 & 1 & 1 & 1 \\
0 & 0 & 1 & 0 & 0 & 0 & 1 \\
0 & 0 & 1 & 1 & 1 & 0 & 1 \\
0 & 1 & 0 & 0 & 1 & 0 & 0 \\
0 & 1 & 0 & 1 & 1 & 0 & 0 \\
0 & 1 & 1 & 0 & 0 & 0 & 1 \\
0 & 1 & 1 & 1 & 0 & 1 & 0 \\
1 & 0 & 0 & 0 & 0 & 0 & 0 \\
1 & 0 & 0 & 1 & 0 & 1 & 1 \\
1 & 0 & 1 & 0 & 0 & 0 & 1 \\
1 & 0 & 1 & 1 & 1 & 1 & 0 \\
1 & 1 & 0 & 0 & 0 & 1 & 1 \\
1 & 1 & 0 & 1 & 0 & 1 & 1 \\
1 & 1 & 1 & 0 & 0 & 0 & 0 \\
1 & 1 & 1 & 1 & 0 & 1 & 0 \\
\hline
\end{tabular}

in no more than $d$ digits from $\vec{x}$, i.e., the distance is $d$ or less. If no such entry exists, $\vec{f}(\vec{x})=0^{m}$. If more than one entry qualifies, the address is determined by identifying all the entries that are the smallest distance from $\vec{x}$ and then by choosing the smallest address from these entries.

Example 2.2 Table 2.3 shows the truth table of the distance 1 CAM function corresponding to the CAM table of Table 2.1. For $\vec{x}=(1,0,0,1)$, there is no entry that matches exactly. However, the entries $(1,1,0,1),(1,0,1,1)$, and $(0,0,0,1)$ at addresses 3,6 , and 7 differ in only one bit. Since the smallest address is $3, \vec{f}(1,0,0,1)=(0,1,1)$.

(End of Example)

\section{$2.3 *$ CAM}

Definition 2.3 An n-input, m-output, p-valued, k-entry *CAM table stores $k$-element $(p+1)$-nary vectors at addresses 1 through $k$. Each $(p+1)$-nary entry consists of $0,1, \ldots, p-1$ and * (don't care). An address is an $m$ element p-nary vector, where $m=\left\lceil\log _{p}(k+1)\right\rceil$. The corresponding *CAM function is a logic function $\vec{f}: P^{n} \rightarrow P^{m}$, where $\vec{f}(\vec{x})$ is the smallest address of an entry that is identical to $\vec{x}$ except for don't care values. If no such entry exists, $\vec{f}(\vec{x})=0^{m}$.

Example 2.3 Table 2.4 shows $a * C A M$ table that stores seven ternary vectors, where $n=4, m=3, p=2$, and $k=7$. The corresponding ${ }^{*} C A M$ function truth table is shown in Table 2.5. The vector $\vec{x}=(1,0,1,1)$ matches the entries at addresses 5 and 6 except for don't care values. Since 5 is smaller, $\vec{f}(1,0,1,1)=(1,0,1)$.

(End of Example)

*CAMs for $p=2$ are extensively used in routing tables for the internet. A routing table specifies an interface identifier corresponding to the longest prefix that matches an incoming packet, in a process called Longest Prefix Match 
Table 2.5. *CAM function truth table

Table 2.4. *CAM

Table

\begin{tabular}{|c|c|}
\hline Address & Vectors \\
\hline 1 & $* 010$ \\
\hline 2 & 0011 \\
\hline 3 & 1101 \\
\hline 4 & 1100 \\
\hline 5 & $* 011$ \\
\hline 6 & $1 * 11$ \\
\hline 7 & $* 001$ \\
\hline
\end{tabular}

tion truth table
\begin{tabular}{|cccc|ccc|}
\hline$x_{1}$ & $x_{2}$ & $x_{3}$ & $x_{4}$ & $f_{2}$ & $f_{1}$ & $f_{0}$ \\
\hline 0 & 0 & 0 & 0 & 0 & 0 & 0 \\
0 & 0 & 0 & 1 & 1 & 1 & 1 \\
0 & 0 & 1 & 0 & 0 & 0 & 1 \\
0 & 0 & 1 & 1 & 0 & 1 & 0 \\
0 & 1 & 0 & 0 & 0 & 0 & 0 \\
0 & 1 & 0 & 1 & 0 & 0 & 0 \\
0 & 1 & 1 & 0 & 0 & 0 & 0 \\
0 & 1 & 1 & 1 & 0 & 0 & 0 \\
1 & 0 & 0 & 0 & 0 & 1 & 0 \\
1 & 0 & 0 & 1 & 1 & 1 & 1 \\
1 & 0 & 1 & 0 & 0 & 0 & 1 \\
1 & 0 & 1 & 1 & 1 & 0 & 1 \\
1 & 1 & 0 & 0 & 1 & 0 & 0 \\
1 & 1 & 0 & 1 & 0 & 1 & 1 \\
1 & 1 & 1 & 0 & 0 & 0 & 0 \\
1 & 1 & 1 & 1 & 1 & 1 & 0 \\
\hline
\end{tabular}

(LPM). In the *CAM table for LPM, the $(p+1)$-nary vectors have restricted patterns: the prefix consists of only 0's, 1 's, ..., and $p-1$ 's, and the postfix consists of only *'s (don't cares).

Definition 2.4 The LPM table stores distinct $(p+1)$-nary vectors of the form $V E C_{1} \cdot V E C_{2}$, where $V E C_{1}$ consists of $O$ 's, l's, ..., and $p-1$ 's, and $V E C_{2}$ consists of *'s. To assure that the longest prefix address is produced, *CAM entries are stored in descending prefix length. The LPM function $i$ s the logic function represented by the *CAM function for the LPM table.

Example 2.4 Consider the LPM table shown in Table 2.6. In the third vector $V E C_{1}=01$ and $V E C_{2}=* *$, while in the last vector $V E C_{1}=0$ and $V E C_{2}=* * *$. Clearly, this is a CAM table for LPM. The truth table for the LPM function is shown in Table 2.7.

(End of Example)

\section{Properties of CAM Functions}

\subsection{C-measure of a Logic Function}

Definition 3.1 Consider a function $\vec{f}(\vec{X}): P^{n} \rightarrow$ $P^{q}$, where $P=\{0,1, \ldots, p-1\}$, and $\vec{X}=\left(x_{1}, x_{2}, \ldots, x_{n}\right)$. Let $\left(\vec{X}_{L}, \vec{X}_{H}\right)$ be a partition of the variable set $\vec{X}$. The decomposition chart for $f$ is a two-dimensional matrix, where the column labels have all possible assignments of values to variables in $\vec{X}_{L}$, the row labels have all possible assignments of values to variables in $\vec{X}_{H}$, and the corresponding matrix value is equal to $\vec{F}\left(\vec{X}_{L}, \vec{X}_{H}\right)$. Among the decomposition charts for $\vec{F}$, the one where $\vec{X}_{L}=\left(x_{1}, x_{2} \ldots, x_{n_{L}}\right)$ and $\vec{X}_{H}=\left(x_{n_{L+1}}, x_{n_{L}+2}, \ldots, x_{n}\right)$ is a standard decomposition chart, where $1 \leq n_{L} \leq n$. The number of different column
Table 2.7. LPM function truth table

Table 2.6. LPM Table

\begin{tabular}{|cccc|ccc|}
\hline$x_{1}$ & $x_{2}$ & $x_{3}$ & $x_{4}$ & $f_{2}$ & $f_{1}$ & $f_{0}$ \\
\hline 0 & 0 & 0 & 0 & 1 & 0 & 1 \\
0 & 0 & 0 & 1 & 1 & 0 & 1 \\
0 & 0 & 1 & 0 & 1 & 0 & 1 \\
0 & 0 & 1 & 1 & 1 & 0 & 1 \\
0 & 1 & 0 & 0 & 0 & 1 & 0 \\
0 & 1 & 0 & 1 & 0 & 1 & 0 \\
0 & 1 & 1 & 0 & 0 & 1 & 1 \\
0 & 1 & 1 & 1 & 0 & 1 & 1 \\
1 & 0 & 0 & 0 & 0 & 0 & 1 \\
1 & 0 & 0 & 1 & 1 & 0 & 0 \\
1 & 0 & 1 & 0 & 1 & 0 & 0 \\
1 & 0 & 1 & 1 & 1 & 0 & 0 \\
1 & 1 & 0 & 0 & 1 & 0 & 0 \\
1 & 1 & 0 & 1 & 1 & 0 & 0 \\
1 & 1 & 1 & 0 & 1 & 0 & 0 \\
1 & 1 & 1 & 1 & 1 & 0 & 0 \\
\hline
\end{tabular}
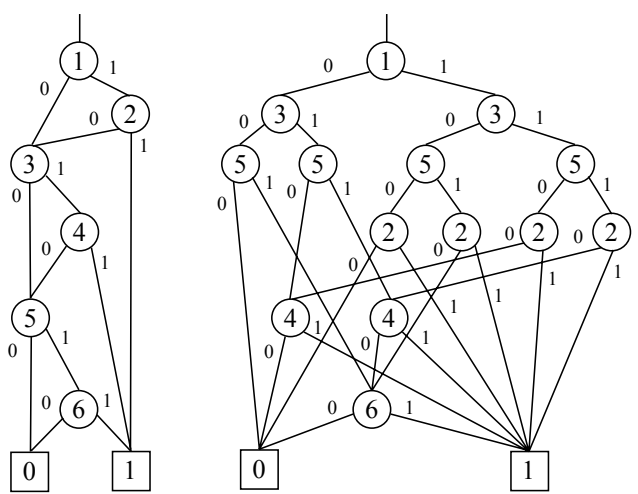

Figure 3.1. BDD for two different orderings.

patterns in the decomposition chart is the column multiplicity of the decomposition chart.

Definition 3.2 The C-measure of a logic function $f$ is the maximum value of all possible column multiplicities of the standard decomposition charts for $f$, where we assume that the ordering of variables is fixed at $\left(x_{1}, x_{2}, \ldots, x_{n}\right)$.

The column multiplicity of a decomposition chart is equal to the width of the MTMDD (multi-terminal multivalued decision diagram). So, the $\mathrm{C}$-measure of a logic function is equal to the maximum width of the MTMDD for the given ordering of the input variables.

Example 3.1 Consider two functions: $f_{1}=x_{1} x_{2} \vee x_{3} x_{4} \vee$ $x_{5} x_{6}$ and $f_{2}=x_{1} x_{5} \vee x_{2} x_{6} \vee x_{3} x_{4}$. Fig. 3.1 shows the BDDs for $f_{1}$ and $f_{2}$. In this case, $C$-measures of $f_{1}$ and $f_{2}$ are 3 and 8 , respectively.

(End of Example)

For a given logic function and variable ordering, the Cmeasure is easy to obtain and uniquely defined. Functions 
having small C-measures have efficient LUT cascade realizations.

Lemma 3.1 For a given function $f$, let $k$ be the number of the input combinations that produce non-zero output values. Then, the C-measure of $f$ is at most $k+1$.

\subsection{CAM}

Theorem 3.1 Consider a CAM table that stores $k$ vectors. The $C$-measure of the CAM function is at most $k+1$.

(Proof) The number of non-zero outputs of the CAM function is at most $k$. From Lemma 3.1, the $\mathrm{C}$-measure is at most $k+1$.

(Q.E.D.)

Theorem 3.1 shows that the worst case value of the Cmeasure is $k+1$. A worst case occurs when the non-zero CAM table entries are widely scattered. However, in practical applications, such as TLBs, the virtual page address will be clustered due to frequent access to the same blocks of memory (e.g., the program or stack). Thus, we expect the actual value of the $\mathrm{C}$-measure to be smaller in practical applications.

\subsection{Distance 1 CAM}

Theorem 3.2 Consider a CAM table that stores $k$ vectors of $n$ digits. Then, the $C$-measure of the distance 1 CAM function is at most $k n(p-1)+k+1$.

(Proof) In the CAM table, for each vector, generate $n(p-$ 1) adjacent vectors, and generate the truth table of the CAM function that shows exact matches and distance 1 matches. Note that the number of non-zero output values of the CAM function is at most $k(n(p-1)+1)$. From Lemma 1, the Cmeasure is at most $k(n(p-1)+1)+1 . \quad$ (Q.E.D.)

\section{$3.4 * \mathrm{CAM}$}

Theorem 3.3 Consider a $*$ CAM table that stores $k$ vectors, where each vector has at most $t$ don't cares. Then, the $C$-measure of the corresponding *CAM function is at most $p^{t} k+1$.

(Proof) In the *CAM table, replace each * by $0,1, \ldots$, and $p-1$, and generate the table without *, i.e., the CAM table. For each pattern, we can generate at most $p^{t}$ vectors. So, the number of non-zero outputs of the *CAM function is at most $p^{t} k$. From Lemma 1, the $\mathrm{C}$-measure is at most $p^{t} k+1$.

(Q.E.D.)

Theorem 3.4 The C-measure of an LPM function with $k$ vectors is at most $k+1$.
(Proof) We prove the theorem for the case where all $k$ entries in the LPM table map to distinct output values. This is the worst case, since forcing certain entries to have the same output value can only reduce the column multiplicity.

We prove the theorem by counting the number of distinct columns in the standard decomposition chart as LPM vectors are added to the LPM table. Reorder the LPM vectors so that those vectors with the most $*$ entries are first and those with the fewest are last. Consider a standard decomposition chart where assignments of values to $x_{1}, x_{2}, \ldots, x_{i}$ label the columns, and assignments of values to $x_{i}, x_{i+1}, \ldots, x_{n}$ label the rows. An "empty" standard decomposition chart has a unique column pattern (all 0's). Let the first vector be $\vec{\alpha}=\left(a_{1}, a_{2}, \ldots, a_{m}, *, *, \ldots, *\right)$, where $a_{j} \in P$. If $m>i$, then the first vector changes only a proper subset of elements in one column. If $m=i(m<i)$, then the new vector changes all elements in one (or more) complete column(s) to the vector's output value in the LPM table. In either case, at most one distinct column pattern is added to the standard decomposition chart.

Because the second vector has no more * entries than the first vector, adding it will change columns only among a subset of the two distinct columns so far in the standard decomposition chart. Let the new vector be $\vec{\beta}=$ $\left(b_{1}, b_{2}, \ldots, b_{m^{\prime}}, *, *, \ldots, *\right)$, where $b_{j} \in P$. If $b_{i}=a_{i}$, for all $1 \leq i \leq m^{\prime}$, then a subset of the columns created by adding $\bar{\alpha}$ to the empty standard decomposition chart are changed. Otherwise, a subset of the columns containing all 0's are changed. In either case, at most one additional column pattern is added. This process continues until all vectors are exhausted. In all, at most $k+1$ column patterns are created. The theorem follows.

(Q.E.D.)

\section{LUT Cascade}

It is possible to realize a CAM function by a standard RAM. For example, the 7-entry CAM shown in Table 2.1 can be realized by a 16-word RAM, where each word has 3 bits, as shown in Table 2.2. The size of the RAM is exponential in the number of bits $n$ used to store the CAM data, even though the CAM contains relatively few data words. The LUT cascade takes advantage of this, offering a way to reduce memory requirements substantially.

Theorem 4.1 For a given function $f$, let $\vec{X}_{L}$ be the column variables, and let $\vec{X}_{H}$ be the row variables, and let $\mu$ be the column multiplicity of the decomposition chart. Then, function $f$ is realizable with the network shown in Fig. 4.1. In this case, the number of p-nary signal lines that connect two blocks $H$ and $G$ is $\left\lceil\log _{p} \mu\right\rceil$.

When the number of $p$-nary signal lines that connect two blocks is smaller than the number of variables in $\vec{X}_{L}$, we can 


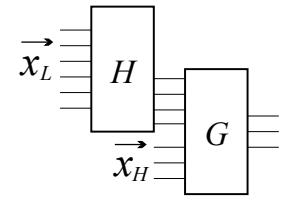

Figure 4.1. Realization of logic functions by decomposition.

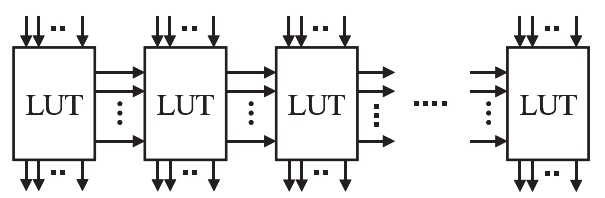

Figure 4.2. LUT cascade with intermediate outputs.

often reduce the size of memory to implement the function. This technique is functional decomposition.

By applying functional decomposition repeatedly to the given function, we have the LUT cascade [11] shown in Fig. 4.2. The cascade consists cells, and the wires connecting adjacent cells are rails. Functions with small Cmeasure require fewer rails, and thus have more compact LUT cascade realizations. To derive C-measures, we need not use decomposition charts. We can efficiently obtain the $\mathrm{C}$-measure from a binary decision diagram (BDD_for_CF) that represents the characteristic function for the multipleoutput function [12].

Theorem 4.2 [11] A logic function with $C$-measure $\mu$ can be implemented by a LUT cascade consisting of cells with at most $\left\lceil\log _{p} \mu\right\rceil+1$ inputs and $\left\lceil\log _{p} \mu\right\rceil$ outputs .

Theorem 4.3 [13] Consider an LUT cascade for a function $f$. Let $n$ be the number of primary inputs, $s$ be the number of cells, $r$ be the maximum number of rails (i.e., the number of lines between cells), $K$ be the maximum number of inputs of a cell, and $\mu$ be the $C$-measure of $f$. If $K \geq\left\lceil\log _{p} \mu\right\rceil+1$, then there is an LUT cascade for $f$ that satisfies the relation:

$$
s \leq\left\lceil\frac{n-r}{K-r}\right\rceil .
$$

\section{Experiment: Two-Valued Case}

We generated random CAM tables, and converted the corresponding CAM functions into MTBDDs.
Table 5.1. C-measure and Size of memory for CAM functions.

\begin{tabular}{|r|c|r|r|r|}
\hline$n$ & $k$ & $\begin{array}{r}\text { \# nodes } \\
\text { MTBDD }\end{array}$ & C-meas & $\begin{array}{r}\text { Mem } \\
\text { M bit }\end{array}$ \\
\hline 32 & 1,000 & 23,156 & 1,001 & 0.44 \\
\hline 32 & 2,000 & 44,316 & 2,001 & 0.94 \\
\hline 32 & 3,000 & 64,628 & 3,001 & 1.88 \\
\hline 32 & 4,000 & 84,512 & 4,001 & 1.88 \\
\hline 32 & 5,000 & 104,125 & 5,001 & 3.75 \\
\hline 32 & 6,000 & 123,408 & 6,001 & 3.86 \\
\hline 32 & 7,000 & 142,402 & 7,001 & 3.86 \\
\hline 32 & 8,000 & 161,237 & 8,001 & 3.86 \\
\hline 32 & 9,000 & 179,879 & 9,001 & 7.41 \\
\hline
\end{tabular}

\subsection{CAM}

For CAM tables, we choose the probability of a 0 and a 1 in the entries to be the same. Table 5.1 shows the result. $n$ denotes the number of variables in the CAM function, and $k$ denotes the number of vectors in the CAM table. $C$ meas shows the $\mathrm{C}$-measure (i.e., the maximum width of the MTBDD). This confirms that the C-measure upper bound (Theorem 3.1) of $k+1$ is firm.

In these example runs, the numbers of CAM table entries are small compared to the total number of possible entries. For example, when $n=32$, the CAM table with $k=1000$ represents $\frac{1000}{2^{32}}=2.3 \times 10^{-7}$ of the total number of entries in the truth table. We expect, therefore, the entries to be widely scattered.

\subsection{Distance 1 CAM}

Tables 5.2 and 5.3 show the results for different numbers of vectors $k$, and different numbers of inputs $n$, respectively.

The expression for the upper bound on the C-measure for distance 1 CAM functions from Theorem 3.2, $n k(p-$ $1)+k+1$, suggests an approximately equal dependence on $n$ and $k$, when $p=2$, and especially when $n$ and $k$ are large. However, in comparing the experimental values for the C-measure shown in Tables 5.2 and 5.3, one sees a much stronger dependence on $k$ than on $n$. That is, Table 5.2, where $n$ is fixed, shows that the C-measure is approximately a linear function of $k$. However, Table 5.3, where $k$ is fixed, shows that the C-measure not strongly dependent $n$.

\section{$5.3 *$ CAM}

\subsubsection{General *CAM Table}

Here, we assume that $p=2$. For $*$ CAM tables, we choose the probability of a 0,1 , and $*$ (don't care) in the entries of the *CAM table to be the same. Table 5.4 shows the 
Table 5.2. C-measure and Size of memory for Distance 1 CAM functions

\begin{tabular}{|r|r|r|r|r|}
\hline$n$ & $k$ & $\begin{array}{r}\text { \# nodes } \\
\text { MTBDD }\end{array}$ & C-meas & $\begin{array}{r}\text { Mem } \\
\text { M bit }\end{array}$ \\
\hline 32 & 100 & 5,751 & 373 & 0.11 \\
\hline 32 & 200 & 11,424 & 801 & 0.25 \\
\hline 32 & 300 & 16,976 & 1,249 & 0.49 \\
\hline 32 & 400 & 22,433 & 1,765 & 0.54 \\
\hline 32 & 500 & 27,997 & 2,260 & 0.76 \\
\hline 32 & 1,000 & 54,931 & 4,867 & 1.64 \\
\hline 32 & 1,500 & 82,020 & 7,610 & 2.31 \\
\hline 32 & 2,000 & 108,700 & 10,363 & 3.38 \\
\hline 32 & 2,500 & 135,340 & 12,975 & 5.34 \\
\hline 32 & 3,000 & 161,352 & 16,048 & 5.81 \\
\hline
\end{tabular}

Table 5.3. C-measure and Size of memory for Distance 1 CAM functions

\begin{tabular}{|r|c|r|r|r|}
\hline$n$ & $k$ & $\begin{array}{r}\text { \# nodes } \\
\text { MTBDD }\end{array}$ & C-meas & $\begin{array}{r}\text { Mem } \\
\text { M bit }\end{array}$ \\
\hline 12 & 500 & 3,903 & 1,457 & 0.04 \\
\hline 14 & 500 & 7,893 & 2,119 & 0.13 \\
\hline 16 & 500 & 11,056 & 2,240 & 0.22 \\
\hline 24 & 500 & 19,858 & 2,260 & 0.54 \\
\hline 32 & 500 & 27,997 & 2,260 & 0.76 \\
\hline 40 & 500 & 35,670 & 2,265 & 0.96 \\
\hline 48 & 500 & 43,807 & 2,271 & 1.18 \\
\hline
\end{tabular}

result. In this case, the $\mathrm{C}$-measure is much greater than in Table 5.1.

In Table 5.4, we take $\delta$ as 11, about one-third of the 32 variables, where $\delta$ is the number of don't cares in the entries. This implies that the number of non-zero outputs for *CAM functions is at most $2^{11}=2048$ times greater than that of CAM functions.

Table 5.4. C-measure and Size of memory for General *CAM functions.

\begin{tabular}{|r|c|r|r|r|}
\hline$n$ & $k$ & $\begin{array}{c}\text { \# nodes } \\
\text { MTBDD }\end{array}$ & C-meas & $\begin{array}{c}\text { Mem } \\
\text { M bit }\end{array}$ \\
\hline 32 & 1,000 & 82,514 & 11,886 & 3.89 \\
\hline 32 & 2,000 & 237,291 & 34,561 & 10.97 \\
\hline 32 & 3,000 & 460,106 & 70,835 & 20.25 \\
\hline 32 & 4,000 & 716,909 & 114,814 & 34.00 \\
\hline 32 & 5,000 & 978,984 & 151,577 & 61.00 \\
\hline 32 & 6,000 & $1,320,175$ & 213,503 & 66.62 \\
\hline 32 & 7,000 & $1,685,556$ & 273,292 & 106.50 \\
\hline 32 & 8,000 & $2,039,238$ & 335,097 & 134.00 \\
\hline 32 & 9,000 & $2,469,748$ & 418,833 & 139.75 \\
\hline
\end{tabular}

Table 5.5. C-measure and Size of memory for LPM functions.

\begin{tabular}{|r|c|r|r|r|}
\hline$n$ & $k$ & $\begin{array}{r}\text { \# nodes } \\
\text { MTBDD }\end{array}$ & C-meas & $\begin{array}{r}\text { Mem } \\
\text { M bit }\end{array}$ \\
\hline 32 & 1,000 & 15,095 & 1,001 & 0.42 \\
\hline 32 & 2,000 & 28,197 & 2,001 & 0.67 \\
\hline 32 & 3,000 & 40,552 & 3,001 & 1.31 \\
\hline 32 & 4,000 & 52,450 & 4,001 & 1.31 \\
\hline 32 & 5,000 & 64,010 & 5,001 & 2.53 \\
\hline 32 & 6,000 & 75,251 & 6,001 & 2.64 \\
\hline 32 & 7,000 & 86,292 & 7,001 & 2.64 \\
\hline 32 & 8,000 & 97,037 & 8,001 & 2.64 \\
\hline 32 & 9,000 & 107,597 & 9,001 & 4.78 \\
\hline
\end{tabular}

Table 6.1. C-measures for Multiple-valued Functions.

\begin{tabular}{|r|r|r|r|r|}
\hline Name & CAM & dist 1 CAM & * CAM & LPM CAM \\
\hline u552 & 548 & 1135 & 553 & 548 \\
\hline u1245 & 1238 & 2528 & 1246 & 1238 \\
\hline u2061 & 2048 & 4286 & 2056 & 2048 \\
\hline u3366 & 3367 & 6891 & 3313 & 3367 \\
\hline
\end{tabular}

\subsubsection{LPM Table}

Here, we assume that $p=2$. In the LPM tables, we generated random prefixes consisting of 0's and 1's. The lengths of the prefixes were chosen from 22 to 26 , and we chose the probability of a 0 and 1 to be the same. Table 5.5 shows that the $\mathrm{C}$-measure is $k+1$. Table 5.5 shows that Theorem 3.4 holds. Compared with the result in [10], the C-measure in our experimental results is about 10 times larger. This is because practical routing tables were used in [10], while we used randomly generated LPM tables.

\section{Experiment: Multi-Valued Case}

For the multiple-valued case, instead of using randomly generated function, we used lists of English words as benchmark functions. We only considered English words with at most 8 letters. For the words with fewer than 8 letters, blanks are appended to the end of the words to make them 8 letters. So, the function has $5 \times 8=40$ input variables. $u 552$ consist of 552 words having 8 letters. $u 1245$ consist of 1245 words having 7 or 8 letters. $u 2061$ consist of 2061 words having 6 or 7 or 8 letters. u3366 consist of 3366 words having 3 to 8 letters. Table 6.1 shows the experimental results for CAMs, distance 1 CAMs, * CAMs, and LPM CAMs.

CAM: For $u 3366$, the C-measure is $k+1=3367$. However, for other functions, the C-measures are slightly 
smaller than $k$.

Distance 1 CAM: The C-measures are approximately $2 k$. The bound given by Theorem 3.2 is $k n(p-1)+k+1 \simeq$ $k(40 \times 25)$, which is much larger than the actual values.

*CAM: To generate random $*$ CAMs, for each word, we replaced just one letter with a "*". The C-measures are always larger than those of corresponding CAMs. The bound given from Theorem 3.3 is $p^{t} k+1=26 \times k+1$, which is much larger than the actual values.

LPM CAM: The C-measures are the same as that of the CAM cases. The bound given from Theorem 3.4 is $k+1$. Note that the exact value matches that of CAMs.

\subsection{LUT Cascade Realization}

Consider the CAM realization of $u 3366$. The C-measure is 3367. Note that $\left\lceil\log _{2} 3367\right\rceil=12$. Thus, by Theorem 4.1, the CAM can be implemented by binary cells with 13 inputs and 12 outputs. By Theorem 4.2, the number of cells is

$$
s=\left\lceil\frac{n-r}{K-r}\right\rceil=\left\lceil\frac{40-12}{13-12}\right\rceil=28,
$$

since $r=\left\lceil\log _{2} 3367\right\rceil=12$, and $K=13$. Thus, the total amount of memory is at most $s \cdot 2^{K} \cdot r=28 \times 2^{13} \times 12=$ $336 \times 2^{13} \simeq 2.7 \times 10^{6}$ bits. Note that the straightforward implementation by a single memory requires $2^{40} \times 12 \simeq$ $1.3 \times 10^{13}$ bits.

\section{Concluding Remarks}

In this paper, we showed a method to implement multiple-valued CAM functions by using LUT cascades. We also defined the C-measure that shows the complexity of LUT cascade. The C-measure is easy to obtain from the MTMDD of the given function. As shown in this paper, LUT cascades are promising for the realization of CAM, LPM, and distance 1 CAM functions. However, an LUT cascade may be too large for the replacement of *CAMs of general functions especially when the ${ }^{*} \mathrm{CAM}$ table has many don't cares.

\section{Acknowledgment}

This work was supported by a grant from the Japanese Ministry of MEXT via Kitakyushu Innovative Cluster Project, the Aid for Scientific Research of the Japan Society for the Promotion of Science (JSPS), and NSA Contract RM A54.

\section{References}

[1] ALTERA, “ Implementing high-speed search applications with Altera CAM," Application Note 119, Altera Corporation.

[2] P. Gupta, S. Lin , and N. McKeown, "Routing lookups in hardware at memory access speeds," Proc. INFOCOM, IEEE Press, Piscataway, N.J., 1998, pp. 1240-1247.

[3] T. Hanyu, N. Kanagawa, and M. Kameyama, "Design of a one-transistor-cell multiple-valued CAM," IEEE Journal of Solid-State Circuits, Vol. 31, No. 11, Nov 1996, pp. 16991674.

[4] M. Kobayashi, T. Murase, and A. Kuriyama, "A longest prefix match search engine for multigigabit IP processing," Proc. Int'l Conf. on Communications (ICC 2000), IEEE Press, Piscataway, N.J., 2000, pp. 1360-1364.

[5] T. Kohonen, Content-Addressable Memories, Springer Series in Information Sciences, Vol. 1, Springer Berlin Heidelberg 1987.

[6] H. Liu, "Routing table compaction in ternary CAM," IEEE Micro, Vol. 22, No.1, Jan.-Feb. 2002, pp. 58-64.

[7] H. J. Mattausch, T. Gyohten, Y. Soda and T. Koide, "Compact associative-memory architecture with fully-parallel search capability for the minimum Hamming distance", IEEE Journal of Solid-State Circuits, Vol.37, No. 2, Feb. 2002, pp. 218-227.

[8] M. Motomura et al., "A 1.2-million-transistor, 33-MHz, 20b dictionary search processor (DISP) ULSI with a 160Kbyte CAM," IEEE J. Solid-State Circuits, Vol. 25, No. 5, Oct. 1990, p. 1158- 1165.

[9] K. Pagiamtzis and A. Sheikholeslami, "A Low-power content-addressable memory (CAM) using pipelined hierarchical search scheme," IEEE Journal of Solid-State Circuits, Vol. 39. No. 9, Sept. 2004, pp.1512-1519.

[10] A. Prakash, R. Kotla, T. Mandal, and A. Aziz, "A highperformance architecture and BDD-based synthesis methodology for packet classification," IEEE Trans. on CAD, Vol. 22, No. 6, pp. 698-709, June 2003.

[11] T. Sasao, M. Matsuura, and Y. Iguchi, "A cascade realization of multiple-output function for reconfigurable hardware," International Workshop on Logic and Synthesis (IWLSO1), Lake Tahoe, CA, June 12-15, 2001, pp. 225-230.

[12] T. Sasao and M. Matsuura, "BDD representation for incompletely specified multiple-output logic functions and its applications to functional decomposition," Design Automation Conference, June 2005, pp.455-462.

[13] T. Sasao, "Analysis and synthesis of weighted-sum functions," IEEE Transaction on CAD, (accepted for publication.)

[14] A. Sheikholeslami, P. G. Gulak, and T. Hanyu, "A multiplevalued ferroelectric content-addressable memory," 26th International Symposium on Multiple-Valued Logic (ISMVL '96), May 1996, pp.74-79.

[15] F. Zane, G. Narlikar, and A. Basu, "CoolCAM: Powerefficient TCAMs for forwarding engines", Proceeding of IEEE INFOCOM '03, April, 2003. 